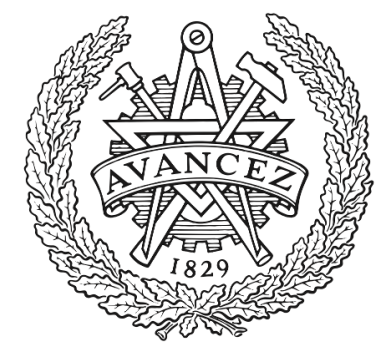

CHALMERS

UNIVERSITY OF TECHNOLOGY

\title{
A GaN-SiC hybrid material for high-frequency and power electronics
}

Downloaded from: https://research.chalmers.se, 2023-04-26 13:41 UTC

Citation for the original published paper (version of record):

Chen, J., Bergsten, J., Lu, J. et al (2018). A GaN-SiC hybrid material for high-frequency and power electronics. Applied Physics Letters, 113(4). http://dx.doi.org/10.1063/1.5042049

N.B. When citing this work, cite the original published paper. 


\title{
A GaN-SiC hybrid material for high-frequency and power electronics
}

\author{
Jr-Tai Chen, ${ }^{1, a)}$ Johan Bergsten, ${ }^{2}$ Jun Lu, ${ }^{3}$ Erik Janzén, ${ }^{1}$ Mattias Thorsell, ${ }^{2}$ Lars Hultman, ${ }^{3}$ \\ Niklas Rorsman, ${ }^{2}$ and Olof Kordina ${ }^{1}$ \\ ${ }^{1}$ SweGaN AB, Teknikringen 8D, Linköping SE583-30, Sweden \\ ${ }^{2}$ Department of Microtechnology and Nanoscience, Chalmers University of Technology, \\ Gothenburg SE412-96, Sweden \\ ${ }^{3}$ Department of Physics, Chemistry, and Biology (IFM), Linköping University, Linköping SE 581 83, Sweden
}

(Received 29 May 2018; accepted 10 July 2018; published online 25 July 2018)

\begin{abstract}
We demonstrate that 3.5\% in-plane lattice mismatch between $\mathrm{GaN}$ (0001) epitaxial layers and $\mathrm{SiC}$ (0001) substrates can be accommodated without triggering extended defects over large areas using a grain-boundary-free AlN nucleation layer (NL). Defect formation in the initial epitaxial growth phase is thus significantly alleviated, confirmed by various characterization techniques. As a result, a high-quality $0.2-\mu \mathrm{m}$ thin GaN layer can be grown on the AIN NL and directly serve as a channel layer in power devices, like high electron mobility transistors (HEMTs). The channel electrons exhibit a state-of-the-art mobility of $>2000 \mathrm{~cm}^{2} / \mathrm{V}$-s, in the $\mathrm{AlGaN} / \mathrm{GaN}$ heterostructures without a conventional thick $\mathrm{C}$ - or Fe-doped buffer layer. The highly scaled transistor processed on the heterostructure with a nearly perfect $\mathrm{GaN}-\mathrm{SiC}$ interface shows excellent $\mathrm{DC}$ and microwave performances. A peak RF power density of $5.8 \mathrm{~W} / \mathrm{mm}$ was obtained at $\mathrm{V}_{\mathrm{DSQ}}=40 \mathrm{~V}$ and a fundamental frequency of $30 \mathrm{GHz}$. Moreover, an unpassivated $0.2-\mu \mathrm{m} \mathrm{GaN} / \mathrm{AlN} / \mathrm{SiC}$ stack shows lateral and vertical breakdowns at $1.5 \mathrm{kV}$. Perfecting the $\mathrm{GaN}-\mathrm{SiC}$ interface enables a $\mathrm{GaN}-\mathrm{SiC}$ hybrid material that combines the high-electron-velocity thin $\mathrm{GaN}$ with the high-breakdown bulk $\mathrm{SiC}$, which promises further advances in a wide spectrum of high-frequency and power electronics. Published by AIP Publishing. https://doi.org/10.1063/1.5042049
\end{abstract}

Structural defects in epitaxial layers dictate semiconductor device performance and reliability. Dislocation annihilation in $\mathrm{GaN}$ is typically a slow process that largely relies on an undesired thick layer growth. ${ }^{1}$ As a wide-bandgap semiconductor, $\mathrm{GaN}$ is being widely used in power devices, yet still suffers from this issue, due to a fundamental heteroepitaxial challenge, the large lattice mismatch to substrates. ${ }^{2,3}$ For GaN-based high electron mobility transistor (HEMT) heterostructures used in high-frequency and power applications, semi-insulating (SI) $\mathrm{SiC}$ remains the best choice of substrates, owing to its high thermal conductivity and high resistivity and the maturity in large-wafer mass production (up to $150 \mathrm{~mm}$ ). Nevertheless, since the first GaN-on-SiC epitaxy was realized by the use of an AlN interlayer more than two decades ago, there has been limited progress in the epitaxial growth. ${ }^{4-7}$ The AlN interlayers/nucleation layers (NLs) typically exhibit grain-like morphology and then evolve into column-like growth as the thickness increases, due to the low mobility of $\mathrm{Al}$ adatoms. Structural defects like voids and dislocations generated at the interfaces of $\mathrm{GaN} / \mathrm{AlN} / \mathrm{SiC}$ introduce a thermal boundary resistance (TBR) that results in an additional $30 \%-40 \%$ channel temperature rise in HEMTs. ${ }^{8}$ Moreover, the threading dislocations in the GaN layer can only be effectively reduced by increasing the thickness, typically to $1.5-2.0 \mu \mathrm{m}$, in order to reach a structural quality sufficient for device performance. Consequently, the thick GaN layer, as a buffer layer, must be doped by acceptor-like impurities such as carbon or iron to eliminate the parasitic conduction below the GaN channel layer, which in turn introduces another grave issue - charge trapping. ${ }^{10-12}$ Charge trapping in deep levels

\footnotetext{
a) Author to whom correspondence should be addressed: jr-tai.chen@swegan.se
}

within the thick buffer layer leads to a current collapse at the device level, which is a major factor limiting the RF output power. Recently, we developed the growth of high-structuralquality AIN NL for low thermal boundary resistance (lowTBR) by hot-wall metalorganic chemical vapor deposition (MOCVD). ${ }^{13,14}$ The typical full width at half maximum (FWHM) value of X-ray diffraction rocking curves (XRC) for the AlN (002) and (102) reflections is around 100 arcsec. In the present work, we additionally find that with the low-TBR AlN NL, the structural quality of a $0.2-\mu \mathrm{m}$-thin unintentionally doped (UID) GaN layer is as mature as that of the typical 2.0- $\mu \mathrm{m}$ thick $\mathrm{GaN}$ layers grown on $\mathrm{SiC}$ substrates. This enables a thin HEMT (T-HEMT) heterostructure, which is based on a concept of a GaN-SiC hybrid material, where the thin $\mathrm{GaN}$ epilayer serves as the high-electron-mobility channel layer and the low-defect bulk $\mathrm{SiC}$ substrate functions as the voltage blocking layer, as proposed in Fig. 1(a). This results in several potential advantages for the T-HEMT heterostructure, compared to traditional HEMT heterostructures with a thick, doped buffer layer: First, the thermal resistance of the T-HEMT devices is expected to be considerably reduced, provided that the formation of structural defects in the $\mathrm{GaN}-\mathrm{SiC}$ interface is suppressed. The joule heat generated in the thin GaN channel would thus dissipate more efficiently into the substrate, so that the issue of device self-heating could be alleviated; Second, by avoiding a thick doped buffer layer, there would be less trapping effects since the entire THEMT heterostructure is unintentionally doped; Third, the AIN NL could effectively serve as a back barrier to enhance the carrier confinement in the channel for high-frequency applications; Finally, the aggressive thickness reduction reduces $90 \%$ raw materials including precursors and gases 


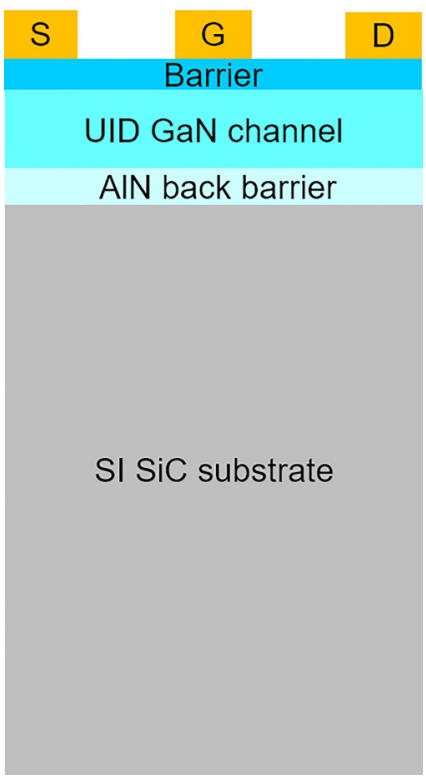

FIG. 1. Schematic thin HEMT (T-HEMT) heterostructure based on the $\mathrm{GaN}-\mathrm{SiC}$ hybrid material concept.

and dramatically reduces the deposition time, thus minimizing the manufacturing cost and increasing MOCVD uptime. All the potential advantages mentioned above were investigated and confirmed in this work.

The III-nitride epitaxial layers were all grown on Si-face $4 \mathrm{H}$ on-axis $\mathrm{SiC}$ substrates in a commercial hot-wall MOCVD reactor using trimethylgallium (TMGa), trimethylaluminum (TMAl), and high-purity ammonia $\left(\mathrm{NH}_{3},>99.999 \%\right)$ as the precursors. The growth was initiated with a normal $60-\mathrm{nm}$ low-TBR AIN NL. On the top of it, the growth was followed by a T-HEMT heterostructure that consists of $2-\mathrm{nm} \mathrm{GaN} \mathrm{cap/}$ 10 14-nm AlGaN barrier/0 1.5-nm AlN/200-nm GaN channel layer. The $\mathrm{Al}$ content in the barrier is around 0.29 0.30. The heterostructure was grown at a high temperature to reduce residual impurities. The levels of $\mathrm{Si}, \mathrm{O}$, and $\mathrm{C}$ are close to the detection limits of secondary ion mass spectroscopy, which is $\sim 1.0 \mathrm{E}+16 \mathrm{~cm}^{-3}$. An interface sharping technique was employed to enhance the transport properties of the channel electrons. The details of the heterostructure growth can be found in Refs. 15 and 16. A high-resolution X-ray diffractometer (Philips X'Pert MRD) with $\lambda=0.154 \mathrm{~nm}$ of $\mathrm{Cu} \mathrm{K}_{\alpha 1}$ radiation was employed to measure the structural parameters of the III-nitride layers. A line focus mode was used, which provides a probe area of $2 \times 5 \mathrm{~mm}^{2}$. An atomic force microscopy (AFM) system (Vecco Dimension 3100) was employed to characterize the sample surface morphology. Transmission electron microscopy (TEM) cross-sectional samples were prepared by ion milling in a Gatan Precision Ion Polishing System using argon ions at $5 \mathrm{kV}$ for $2 \mathrm{~h}, 2 \mathrm{kV}$ to electron transparent, and finally polishing at $500 \mathrm{~V}$ for $2 \mathrm{~h}$. FEI Tecnai G2 TF 20 UT with a field-emission gun operated at $200 \mathrm{keV}$ and a point resolution of $1.95 \AA$ was used for TEM characterization.

We examined the properties of a thin HEMT heterostructure for a model high-frequency power device, consisting of 2$\mathrm{nm}$ GaN cap/14-nm $\mathrm{Al}_{0.29} \mathrm{Ga}_{0.71} \mathrm{~N}$ barrier layer/200-nm GaN channel layer/60-nm low-TBR AlN NL grown on a $100 \mathrm{~mm}$ semi-insulating (SI) $4 \mathrm{H} \mathrm{SiC}$ substrate by using several material (a)

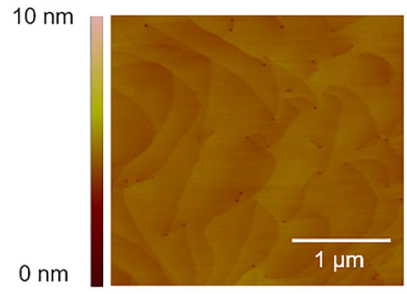

(c)

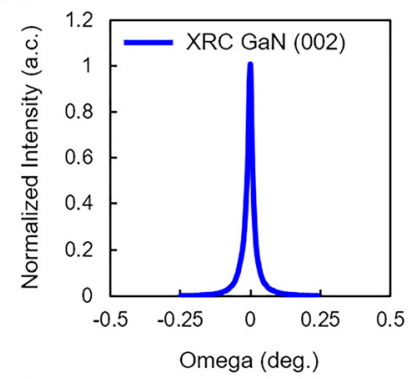

(d)

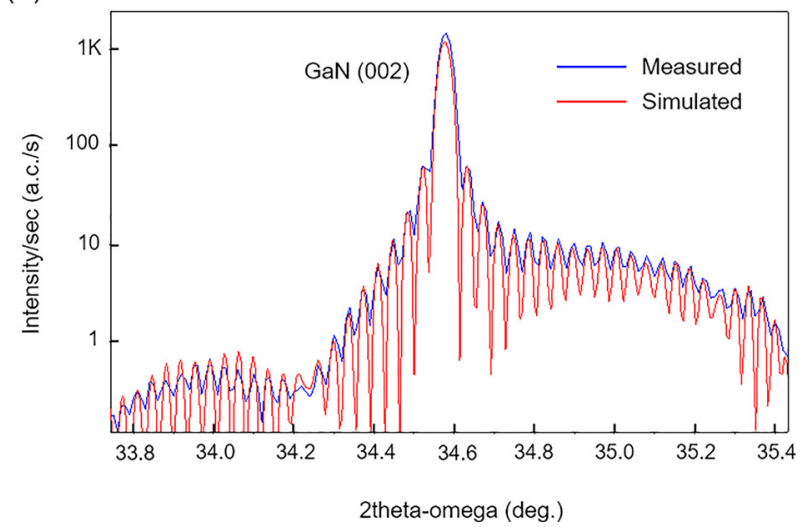

(b)
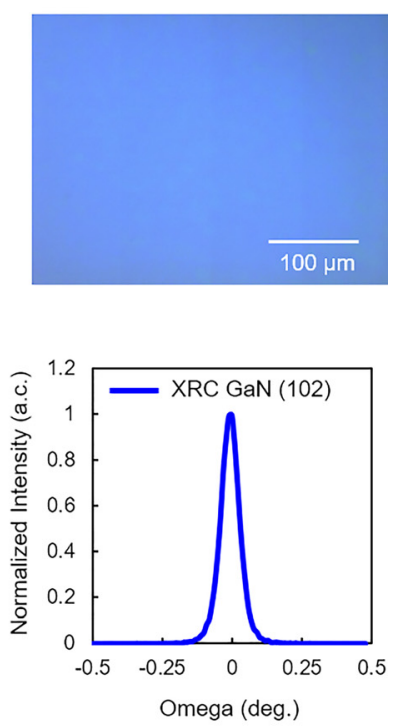

FIG. 2. (a) $3 \times 3 \mu \mathrm{m}^{2}$ AFM image, (b) OM image, (c) GaN XRCs of the T-HEMT, and (d) XRD 2theta-omega of the thin GaN (002).

characterization techniques. Figures 2(a) and 2(b) show the images of a small and a large surface area by atomic force microscopy (AFM) and Nomarski optical microscopy $(\mathrm{OM})$, respectively. A root-mean-square surface roughness of $0.235 \mathrm{~nm}$ was measured over a $3 \times 3 \mu \mathrm{m}^{2}$ area. Both images indicate that the heterostructure has a fully coalesced and atomically flat morphology. Furthermore, the crystalline quality of the GaN layer was evaluated by X-ray diffraction rocking curve measurements for the (002) and (102) reflections to assess screw-type and edge-type dislocations, respectively. As shown in Fig. 2(c), the thin GaN channel layer exhibits a full width at half maximum of 86 and 268 arcsec for the GaN XRC (002) and (102) reflections, respectively. The threading dislocation density in the $\mathrm{GaN}$ layer is estimated to be in the range of low $10^{8} \mathrm{~cm}^{-2}$, based on the FWHMs of the XRCs. ${ }^{17}$ This defect density is two orders of magnitude less than that of the typical GaN layers with the same thickness. ${ }^{1}$ Besides, in Fig. 2(d), one can see that the thin GaN layer exhibits strong Kiessig fringes, from which a channel thickness of $220 \mathrm{~nm}$ is extracted. It should be noted that this feature is pronounced only when the thin layer has a high-quality and sharp heterojunctions. Contactless Hall measurements revealed that the epiwafer has a two-dimensional electron gas (2DEG) density of $9.8 \times 10^{12} \mathrm{~cm}^{-2}$ and a 2 DEG 
(a)

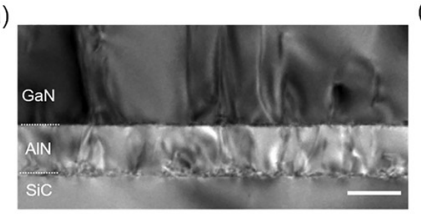

(b)

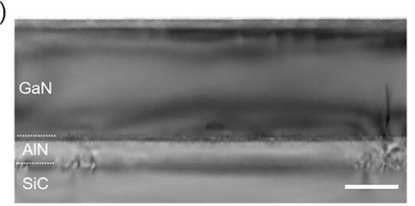

(c)

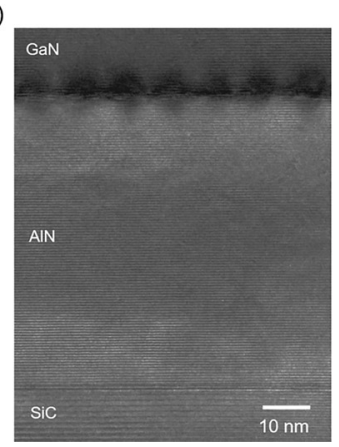

(d)

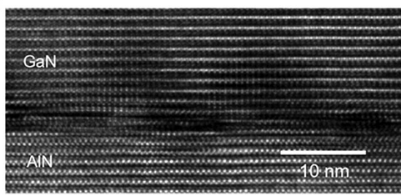

(e)

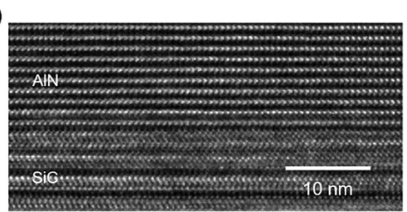

FIG. 3. Cross-sectional TEM images along $\left[\begin{array}{lll}11 & \overline{2} & 0\end{array}\right]$ at the $\mathrm{GaN} / \mathrm{AlN} / \mathrm{SiC}$ interface using (a) a conventional AIN NL and (b) the low-TBR AlN NL. (c) High-magnification image of GaN/ low-TBR AlN NL/SiC. (d) Highresolution image at the interface of GaN/low-TBR AlN NL. (e) Highresolution image at the interface of low-TBR AlN NL/SiC. The scale bar is $100 \mathrm{~nm}$ in (a) and (b). mobility of $2050 \mathrm{~cm}^{2} / \mathrm{V}$-s, which results in a sheet resistance $\left(\mathrm{R}_{\mathrm{sh}}\right)$ of $315 \mathrm{ohm} / \mathrm{sq}$. The uniformity of the $\mathrm{R}_{\mathrm{sh}}$ measuring 17 points over the $100 \mathrm{~mm}$ epiwafer is $1.8 \%$ (S1). All the structural and electrical properties achieved above are unprecedented for such a thin III-nitride HEMT heterostructure. It is also worth emphasizing that these properties are on par with those of the state-of-the-art GaN-on-SiC epiwafers with the conventional thick buffer layers. To gain further insights into the epitaxy, the high-resolution TEM was deployed to investigate the $\mathrm{GaN}-\mathrm{SiC}$ interface.

Figures 3(a) and 3(b) show cross-sectional TEM images in the GaN/AlN/SiC interface region of the HEMT heterostructures using a conventional AlN NL ${ }^{15}$ and our here presented low-TBR AIN NL, respectively. It confirms that the low-TBR AIN NL has a very high structural integrity and is visibly free of grain boundaries, as highlighted in Fig. 3(c). Large defect-free regions up to $300 \sim 400 \mathrm{~nm}$ wide are dominant in the low-TBR AIN NL, which is in stark contrast to the conventional AIN nucleation layer that has a high grain/ defect density. The noticeable defects in the low-TBR AIN $\mathrm{NL}$ actually originate from the $\mathrm{SiC}$ surface region. This shows that besides the AlN NL growth, the surface condition of the $\mathrm{SiC}$ and its crystalline quality are also of importance to minimize the defect formation. Moreover, a closer look at the GaN/AlN interface and the AlN/SiC interface, as shown in Figs. 2(d) and 2(e), respectively, reveals that the lattice mismatch between $\mathrm{GaN}$ and $\mathrm{SiC}$ can be well accommodated with the periodic misfits at the GaN/AlN interface, given that part of the strain is released inevitably through the generation of threading dislocations. The reason that the periodic misfits only appear at the GaN/AlN interface, not at the AlN/SiC interface, is likely due to the fact that the lattice mismatch of the former interface $(2.5 \%)$ is much larger than that of the latter $(1 \%)$. On the other hand, this result also suggests that the formation of threading dislocations can be considerably suppressed in the initial epitaxial growth through the formation of the periodic misfits.

The test transistor devices were fabricated on a T-HEMT heterostructure consisting of similar active layers (2-nm GaN cap/10-nm $\mathrm{Al}_{0.30} \mathrm{Ga}_{0.70} \mathrm{~N}$ barrier layer/1-nm AlN/0.2- $\mu \mathrm{m} \mathrm{GaN} \mathrm{channel)} \mathrm{with} \mathrm{gate} \mathrm{lengths} \mathrm{of} \mathrm{0.1-} \mathrm{and} \mathrm{0.2-}$ $\mu \mathrm{m}$ and a source-drain spacing of $2.0 \mu \mathrm{m}$. The details of the device processing for the AlGaN can be found in Ref. 18. A contact resistance of $0.3 \Omega \mathrm{mm}$ was obtained using recessed Ta-based contact. ${ }^{19}$

Figure 4(a) shows the DC I-V characteristic of the $0.1 \mu \mathrm{m}$ T-HEMT device measured using a parameter analyzer (Keithley 4200-SCS). The device exhibited a high current density of $1.1 \mathrm{~A} / \mathrm{mm}$ and a low on-resistance of $1.3 \Omega$ $\mathrm{mm}$. It was noticed that the high saturation current can be sustained at a $\mathrm{V}_{\mathrm{DS}}$ up to $30 \mathrm{~V}$ without a noticeable degradation due to self-heating. This confirms the merit of the thermal management in the thin heterostructure. Moreover, the transfer characteristics at $\mathrm{V}_{\mathrm{DS}}=10 \mathrm{~V}$ in Figs. 4(b) and 4(c) show that the device has a sharp pinch-off and a very high transconductance of $500 \mathrm{mS} / \mathrm{mm}$. The subthreshold swing (SS) was estimated to be 250 and $130 \mathrm{mV} /$ decade at $\mathrm{V}_{\mathrm{DS}}$ $=10 \mathrm{~V}$, for the $0.1-$ and $0.2-\mu \mathrm{m}$ T-HEMT devices, respectively. These results indicate that the channel electrons are (a)

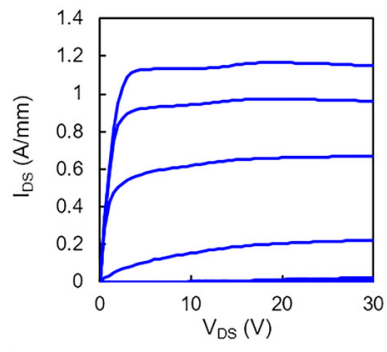

(c)

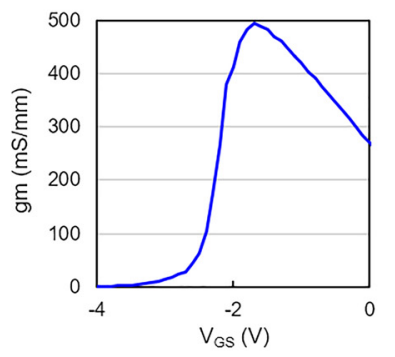

(b)

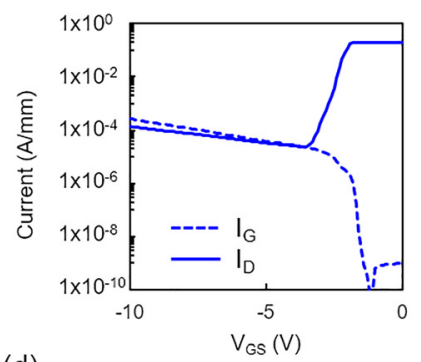

(d)

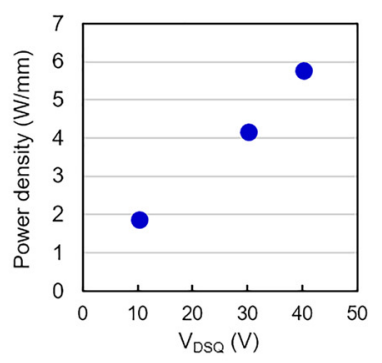

(e)

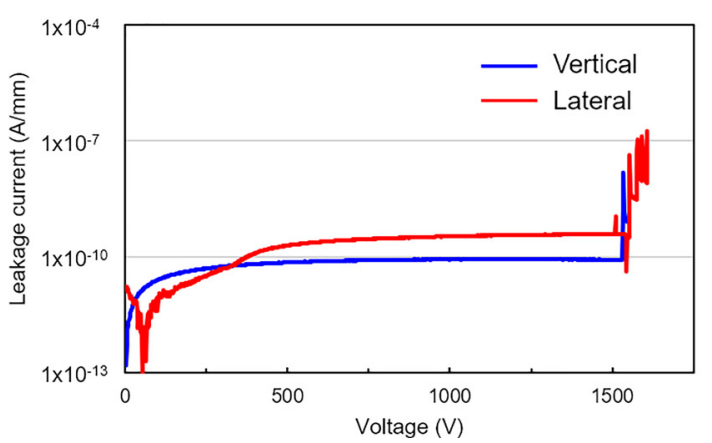

FIG. 4. (a) DC I-V characteristics, (b) transfer characteristics and gate and drain current at $\mathrm{V}_{\mathrm{DS}}=10 \mathrm{~V}$ as a function of gate voltage, (c) transconductance as a function of $\mathrm{V}_{\mathrm{GS}}$, and (d) RF output power density as a function of $\mathrm{V}_{\text {DSQ }}$ of the T-HEMT device. (e) Vertical and lateral breakdown characteristics of the T-HEMT heterostructure without the top active layers. 
well-confined in the T-HEMT heterostructures assisted by the AIN NL as a back barrier, which is essential for highfrequency applications. Meanwhile, the measured breakdown voltage of the $0.1-$ and $0.2-\mu \mathrm{m}$ T-HEMT devices is 70 and $140 \mathrm{~V}$, respectively. The linear relationship between the breakdown voltage and the gate length suggests that the breakdown was taking place laterally due to the limited dimension of the gate length and the gate-to-drain spacing. The typical gate length and the gate-to-drain spacing used in lateral power devices are in the ranges of $1 \sim 2 \mu \mathrm{m}$ and $10 \sim 20 \mu \mathrm{m}$, respectively. Therefore, this result reveals the great potential of the T-HEMTs for lateral power devices. ${ }^{3}$

The microwave power performance was evaluated through continuous wave (CW) large signal measurements using a passive load-pull setup at a fundamental frequency of $30 \mathrm{GHz}$. The output power density of the $0.1-\mu \mathrm{m} \mathrm{T}$ HEMT was measured with a quiescent current of $15 \%$ of the saturation current and biased at $\mathrm{V}_{\mathrm{DSQ}}=10,30$, and $40 \mathrm{~V}$, respectively. The output power density as a function of the bias is plotted in Fig. 4(d). It is obvious that the T-HEMT is capable of delivering very high RF power. A peak RF power density of $5.8 \mathrm{~W} / \mathrm{mm}$ was obtained at $\mathrm{V}_{\mathrm{DSQ}}=40 \mathrm{~V}$. We attribute the high RF power to the absence of the intentional acceptor-like dopant in the T-HEMT heterostructure. Without the need to grow a thick doped buffer layer, the T-HEMT heterostructure opens up a broad opportunity to further reduce charge trapping effects.

As sparked by the previous breakdown result, we additionally measured another sample to further explore the potential of T-HEMT heterostructures for lateral power devices. The sample has the same T-HEMT heterostructure but without the top active layers, i.e. a stack of $0.2-\mu \mathrm{m} \mathrm{GaN}$ channel/60-nm AlN NL/500- $\mu \mathrm{m} \mathrm{SiC} \mathrm{substrate.} \mathrm{Figure} \mathrm{4(e)}$ shows the two-terminal breakdown characteristics of the sample, measured laterally with the substrate floating (the two-terminal spacing is $\sim 1.5 \mathrm{~mm}$ ) and vertically with the substrate grounded, at room temperature. Surprisingly, the breakdown voltage in both the lateral and vertical configurations is as high as $\sim 1.5 \mathrm{kV}$ for the stack containing such a thin GaN layer. In both cases, the breakdown was due to the bad delineation of the contacts. Therefore, the real breakdown voltage of the stack is expected to be higher. In other words, the breakdown has been limited by the surface and it confirms that there are no interface carriers. To obtain this high vertical breakdown voltage, the SI SiC substrate must be effectively acting as a voltage blocking layer as we proposed earlier. This can be understood from GaN-on-Si lateral power devices, ${ }^{20}$ in which it has been evident that the device breakdown is limited vertically. This is why the breakdown voltage can increase from $200 \mathrm{~V}$ to $650 \mathrm{~V}$ as the buffer layer thickness increases from $3.2 \mu \mathrm{m}$ to $5.5 \mu \mathrm{m}$, and the breakdown voltage is further increased if the $\mathrm{Si}$ substrate is removed. These results indicate that the thick buffer layer and the Si substrate are the breakdown-limiting places, not the surface. Meanwhile, we noticed that there is a leakage current dip at $\sim 60 \mathrm{~V}$ in the lateral breakdown test. The mechanism is unclear at the moment. Albeit that a detailed study of the T-HEMT heterostructure for lateral power devices is still ongoing, the result of the vertical breakdown voltage of $1.5 \mathrm{kV}$ has shined the light on the future of T-HEMT heterostructures for lateral power devices. With proper surface passivation, it holds promise to block substantially higher voltage.

In summary, we report that a nearly perfect $\mathrm{GaN}-\mathrm{SiC}$ interface can be realized by using a grain-boundary-free AIN NL, which enables us to remove the conventional thick C- or $\mathrm{Fe}$ - doped buffer layer in GaN-based HEMT heterostructures. This provides alternative routes to tackle the buffertrapping issues that have been prevailing in the field over the last two decades. The excellent DC and microwave device characteristics plus the extraordinary high breakdown confirm the competence of the proposed T-HEMT heterostructure that is based on the $\mathrm{GaN}-\mathrm{SiC}$ hybrid material concept, for the use in both high-frequency and power applications.

See supplementary material for the $\mathrm{R}_{\text {sh }}$ map of the $\mathrm{T}$ HEMT heterostructure grown on a SI $100 \mathrm{~mm} \mathrm{SiC} \mathrm{substrate.}$

${ }^{1}$ S. K. Mathis, A. E. Romanov, L. F. Chen, G. E. Beltz, W. Pompe, and J. S. Speck, "Modeling of threading dislocation reduction in growing GaN layers," Phys. Status Solidi A 179, 125 (2000).

${ }^{2}$ F. A. Marino, N. Faralli, T. Palacios, D. K. Ferry, S. M. Goodnick, and M. Saraniti, "Effects of threading dislocations on AlGaN/GaN high-electron mobility transistors," IEEE Trans. Electron Devices 57, 353 (2010).

${ }^{3}$ M. Ishida, T. Ueda, T. Tanaka, and D. Ueda, "GaN on Si technologies for power switching devices," IEEE Trans. Electron Devices 60, 3053 (2013).

${ }^{4}$ F. A. Ponce, B. S. Krusor, J. S. Major, Jr., W. E. Plano, and D. F. Welch, "Microstructure of GaN epitaxy on SiC using AlN buffer layers," Appl. Phys. Lett. 67, 410 (1995).

${ }^{5}$ B. Morana, F. Wu, A. E. Romanov, U. K. Mishra, S. P. Denbaars, and J. S. Speck, "Structural and morphological evolution of GaN grown by metalorganic chemical vapor deposition on $\mathrm{SiC}$ substrates using an AlN initial layer," J. Cryst. Growth 273, 38 (2004).

${ }^{6}$ Z. J. Reitmeier, S. Einfeldt, R. F. Davis, X. Zhang, X. Fang, and S. Mahajan, "Surface and defect microstructure of GaN and AlN layers grown on hydrogen-etched 6H-SiC (0001) substrates," Acta Mater. 58, 2165 (2010).

${ }^{7}$ E. Cho, A. Mogilatenko, F. Brunner, E. Richter, and M. Weyers, "Impact of AlN nucleation layer on strain in GaN grown on $4 \mathrm{H}-\mathrm{SiC}$ substrates," J. Cryst. Growth 371, 45 (2013).

${ }^{8}$ A. Sarua, H. Ji, K. P. Hilton, D. J. Wallis, M. J. Uren, T. Martin, and M. Kuball, "Thermal boundary resistance between GaN and substrate in AlGaN/GaN electronic devices," IEEE Trans. Electron Devices 54, 3152 (2007).

${ }^{9}$ A. Manoi, J. W. Pomeroy, N. Killat, and M. Kuball, "Benchmarking of thermal boundary resistance in AlGaN/GaN HEMTs on SiC substrates: Implications of the nucleation layer microstructure," IEEE Trans. Electron Devices 31, 1395 (2010).

${ }^{10}$ E. Bahat-Treidel, F. Brunner, O. Hilt, E. Cho, J. Wurfl, and G. Trankle, "AlGaN/GaN/GaN:C back barrier HFET with breakdown voltage," IEEE Trans. Electron Devices 57, 3050 (2010).

${ }^{11}$ S. Heikman, S. Keller, S. P. DenBarrs, and U. K. Mishira, "Growth of Fe doped semi-insulating GaN by metalorganic chemical vapor deposition," Appl. Phys. Lett. 81, 439 (2002).

${ }^{12}$ M. J. Uren, J. Möreke, and M. Kuball, "Buffer Design to minimize current collapse in GaN AlGaN HFETs," IEEE Trans. Electron Devices 59, 3327 (2012).

${ }^{13}$ J.-T. Chen, J. W. Pomeroy, N. Rorsman, C. Xia, C. Virojanadara, U. Forsberg, M. Kuball, and E. Janzén, J. Cryst. Growth 428, 54 (2015).

${ }^{14}$ J.-T. Chen, E. Janzén, N. Rorsman, M. Thorsell, M. Andersson, and O. Kordina, "Carbon-doped $\mathrm{GaN}$ on $\mathrm{SiC}$ materials for low-memory-effect devices," ECS Trans. 75, 61 (2016).

${ }^{15}$ J.-T. Chen, U. Forsberg, and E. Janzén, "Impact of residual carbon on twodimensional electron gas properties in AlGaN/GaN heterostructure," Appl. Phys. Lett. 102, 193506 (2013).

${ }^{16}$ J.-T. Chen, I. Persson, D. Nilsson, C.-W. Hsu, J. Palisaitis, U. Forsberg, P. O. A. Persson, and E. Janzén, "Room-temperature mobility above 
$2200 \mathrm{~cm} 2 / \mathrm{V} \cdot \mathrm{s}$ of two-dimensional electron gas in a sharp-interface AlGaN/GaN heterostructure," Appl. Phys. Lett. 106, 251601 (2015).

${ }^{17}$ B. Heying, X. H. Wu, S. Keller, Y. Li, D. Kapolnek, B. P. Keller, S. P. DenBaars, and J. S. Speck, "Role of threading dislocation structure on the x-ray diffraction peak widths in epitaxial GaN films," Appl. Phys. Lett. 68(5), 643 (1996).

${ }^{18}$ J. Bergsten, J.-T. Chen, S. Gustafsson, A. Malmros, U. Forsberg, M. Thorsell, E. Janzén, and N. Rorsman, "Performance enhancement of microwave GaN HEMTs without an AlN-exclusion layer using an optimized AlGaN/GaN interface growth process," IEEE Trans. Electron Devices 63, 333 (2016).

${ }^{19}$ A. Malmros, H. Blanck, and N. Rorsman, "Electrical properties, microstructure, and thermal stability of Ta-based ohmic contacts annealed at low temperature for GaN HEMTs," Semicond. Sci. Technol. 26, 075006 (2011).

${ }^{20}$ H. Amano, Y. Baines, E. Beam, M. Borga, T. Bouchet, P. R. Chalker, M. Charles, K. J. Chen, N. Chowdhury, R. Chu et al., "The $2018 \mathrm{GaN}$ power electronics roadmap," J. Phys. D: Appl. Phys. 51, 163001 (2018). 